\title{
INVESTIGATING THE RHYTHMS OF HUMAN MOVEMENTS IN GENEVA LAKE REGION USING MDC DATA
}

\author{
R. Javanmard*, R. Esmaeili, , M. Malekzadeh, F. Karimipour
}

School of Surveying and Geospatial Engineering, College of Engineering, University of Tehran, Tehran, Iran - (reyhanejavanmard, roya.esmaeili, miladmalekzadeh, fkarimipour)@ut.ac.ir

\section{Commission VI, WG VI/4}

KEY WORDS: Movement pattern, Demographic Variables, time variable

\begin{abstract}
:
Movement data are becoming extensive and comprehensive with the advent of GPS (global positioning system) and pervasive use of smartphones, which has led to an increasing rate of studies about movement such as mobility pattern of oil spills, taxies, storms and animals. Studying the movement of people has long been the topic of much thought and debate among researchers within the field of transportation, social issues, and policy. One of the basic prerequisites for studying human movement behavior is modeling the movement, which show how people move so that the effect of different variables can be revealed. For this purpose, this research intends to deploy the concept of activity space (i.e., the part of the space in which a person is active) and its determinants to display the trajectory of individuals, and then modeling the effect of different variables on human mobility behavior. This study explores the effect of time (movement on weekends and weekdays) and demographic (age, gender, occupation state) factors on the characteristics of human mobility pattern and analyzes the extent to which the mobility pattern of different group of people is related to time by using Swiss human movement sample dataset, called MDC. These movement characteristics can be used later in a wide range of applications, such as predictions, urban planning, and traffic forecasting.
\end{abstract}

\section{INTRODUCTION}

Human mobility plays an important role in the study of traffic forecasting, disease spreading, urban planning, and in the general science and engineering of smart cities (Luo, Cao et al. 2016). In the past, most of the efforts to understand the human movement were based on the data collected by questionnaires (Yamamoto and Kitamura 1999), which were expensive, not big enough and led to a time-consuming, inefficient process. However, the amelioration of Web and mobile technologies yields a set of reliable and cost-effective data sources that has triggered studies of human mobility patterns (Shi, Chi et al. 2015).

Human mobility is restricted by a multitude of demographic factors such as age, gender, occupation, and income. In addition, temporal order (e.g., weekends and weekdays) are considered to have an important impact on individual movement behavior (Yuan, Raubal et al. 2012). Due to the inherent inconsistency of movement patterns on weekdays and weekends, it is necessary to explore human mobility under different temporal orders.

This paper aims to explore the correlation between demographic variables (age, gender, and occupation) and human movement pattern with time perspective by exploiting Swiss human movement sample dataset, i.e., Mobile Data Challenge (MDC) dataset, in Geneva Lake Region.

\section{METHODOLOGY}

Activity space is defined as a geographic coverage of an individual's movement trajectory by considering visited places and taken routes that helped her get to her destinations (Lee,
Voss et al. 2016). There are several related concepts, such as the action space (Horton and Reynolds 1971), the awareness space (Brown and Moore 1970), or space-time prisms (Hagerstrand 1970)

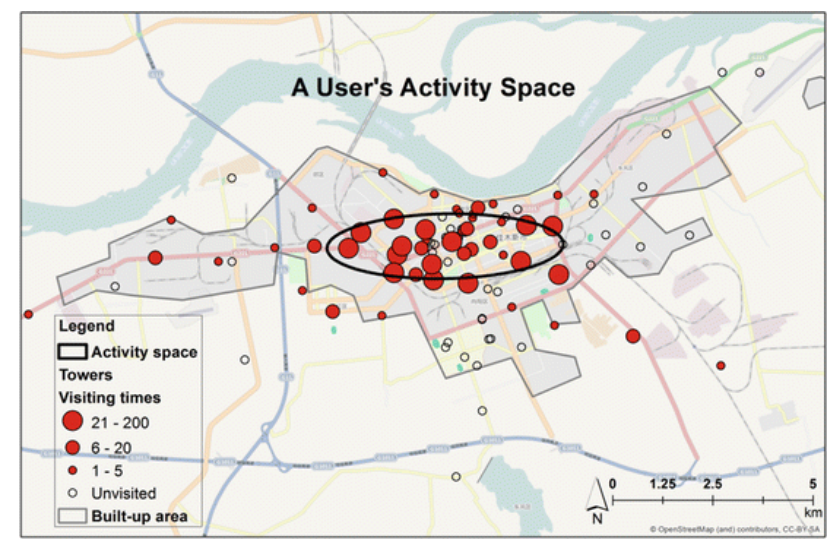

Figure 1. deviational ellipse (Wang, Kang et al. 2015)

The approximation and measurement of activity space depict the basic characteristics of activity space (size, shape, etc.). Historically, activity space is measured based on two types of methods: Eclipse-based representation (Figure 1), e.g., standard deviational ellipse, the radius of gyration (Song, Qu et al. 2010) and network-based representation (e.g., road networks) (Sherman 2011). We adopt the ellipse-based method to better characterize the geometric properties of activity spaces. In order to identify the characteristics of activity space, the following determinants have been employed:

\footnotetext{
* Corresponding author
} 
Area: It indicates the extent of the activity space (Newsome, Walcott et al. 1998), and is used to depict the dispersion of the visited locations (Schönfelder and Axhausen 2003). The area of the standard deviation ellipse is calculated as follow:

$$
\text { area }=\pi a b
$$

where $\mathrm{a}$ is the semi-major axis and $\mathrm{b}$ is the semi-minor axis of the ellipse.

Shape index (1-eccentricity): As users' trajectories are approximated as ellipses, the movement eccentricity represents how much a particular activity space's shape deviates from a circular shape:

$$
e=\sqrt{1-\left(\frac{b}{a}\right)^{2}}
$$

For instance, if $e \approx 1$, it is highly possible that the particular person mostly moves between popular pegs of her life such as work and home; therefore, the shape of activity space is close to a regular straight line rather than a circle (Yuan and Raubal 2016). Here, we use 1-eccentricity to represent the extent to which an activity space deviates from a straight line. The higher the value of shape index, the more scattered is a user's visited places and the more is the shape of her activity space like a circle rather than a thin ellipse.

\section{RESULTS}

\subsection{Dataset}

In January 2009, Nokia Research Centre Lausanne and its Swiss academic partners designed the Lausanne Data Collection Campaign (LDCC) to create large-scale mobile data researcher sources. Data was collected using Nokia N95 phones and around 185 volunteers (38\% male and $62 \%$ female) who lived near Geneva Lake contributed in this project over a period of one year. Although myriad types of data were recoded from the smartphones of volunteers, this paper has used demographic information of participants (age, gender, and the occupation), and their location information produced by cell phone's GPS (time, longitude, latitude) in every 5 seconds (Kiukkonen, Blom et al. 2010, Laurila, Gatica-Perez et al. 2012).

In the following section, users' trajectories are separated based on the days of the week and then the determinants of the activity space are calculated for each individual. Subsequently, the average and standard deviation of the mentioned parameters in each group is used for detecting the impact of time on users' movement.

\subsection{Correlation between days of the week and activity space}

Isaacman deployed median daily range and maximum daily range to understand the effect of time on the movement of people in America using mobile phone data (Isaacman, Becker et al. 2011). In this research, activity space is fitted to the maximum travel distance of some users in Switzerland instead of the median of the daily range.

According to the area of activity spaces (Tables 1, 2 and Figure 2 ), users have on weekends bigger activity spaces than on weekdays. It shows that users tend to travel to further places on weekends, which could be due to the closure of schools and having more free time during weekends. In addition, users have a higher standard deviation of the area on weekends that indicates bigger diversities among users' movement behavior on weekends (Table 2). One reason of this difference might be stemmed from the idea that the majority of users tend to spend their weekend at home but those who travel are eager to go to further and scattered destinations (Isaacman, Becker et al. 2011). However, in contrast to Yuan's results in 2013, the difference between the shape index of the users' activity space on weekends and weekdays is not too much (Yuan 2013). Note that general holidays are omitted from Table 1 and 2.

\begin{tabular}{|c|c|c|c|c|c|}
\hline Days of the week & $\mathbf{N o}^{\mathbf{1}}$. users & $\mathbf{A v g}^{\mathbf{2}}$ area $^{\mathbf{3}}$ & $\mathbf{S t d}^{\mathbf{3}}$ area & Avg SI & Std SI $^{\mathbf{4}}$ \\
\hline Saturday & 152 & 1206356320 & 1735113197 & 0.1108 & 0.1127 \\
Sunday & 150 & 1230619443 & 1812527016 & 0.1282 & 0.1355 \\
Monday & 150 & 540205863 & 1132220687 & 0.085 & 0.1128 \\
Tuesday & 152 & 610718147 & 1329976328 & 0.1016 & 0.129 \\
Wednesday & 153 & 565862949 & 946240586 & 0.0883 & 0.1014 \\
Thursday & 152 & 519474182 & 768785652 & 0.099 & 0.116 \\
Friday & 152 & 760972719 & 1247755746 & 0.1099 & 0.1253 \\
\hline
\end{tabular}

Table 1. Average and standard deviation of area and shape index of users' activity space grouped by days of the week

\begin{tabular}{|c|c|c|c|c|c|}
\hline Week & No. users & Avg area & Std area & Avg SI & Std SI \\
\hline weekends & 152 & 1412443579 & 1790098291 & 0.1357 & 0.123 \\
weekdays & 155 & 784907146 & 1041589367 & 0.1219 & 0.124 \\
\hline
\end{tabular}

\footnotetext{
${ }^{1}$ Number of

2 Average of

${ }^{3}$ Standard deviation of

${ }^{4}$ Shape index
} 
Table 2. Average and standard deviation of area and shape index of users' activity space grouped by weekdays and weekends

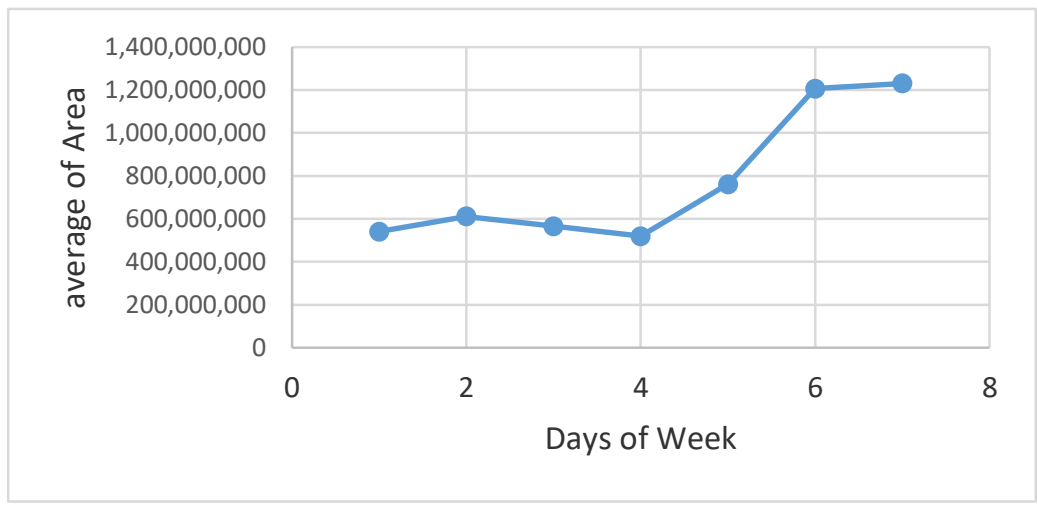

Figure 2. The relation between days of the week and users' activity space

Many other variables could affect the relationship between time and the determinants of activity space. For example, as Crane showed, having a car, job position, family and marital status, etc., have an impact on the relationship between gender and determinants of activity space (Crane 2007). Therefore, in the following sections, in order to investigate the relationship between demographic variables (gender, age, and occupation), days of the week and users' movement behavior, users in the MDC dataset were first classified with regard to their gender, age, and occupation. Then the effect of time on the determinants of the activity space of individuals' trajectories in each demographic group was analyzed.

\subsection{Correlation between gender, days of the week and activity space}

As seen in Tables 3, by calculating the parameters of activity space for each user in male and female group and then averaging them, it is determined that both men and women have higher values for their activity space area and shape index on weekends, which demonstrate that people on weekends, have a longer distance travels, visit more scattered places, and shape of their activity space is far from the straight line. However, with regard to Table 4 and the greater standard deviation of the average of men's activity space determinants, the impact of time (days of the week) on their movement behavior is much higher than that of women. Men experience further and more dispersed travels during weekends. Moreover, having greater standard deviation of activity space's determinants shows that the difference in their mobility behaviour is much higher on weekends compared to women. This difference could be attributed to the fact that most of men tend to spend their weekend near their home, but those minorities who travel have very long journeys with scattered destinations.

\begin{tabular}{|c|c|c|c|c|c|c|}
\hline gender & Week (female) & No. users & Avg area & Std area & Avg SI & Std SI \\
\hline \multirow{2}{*}{ Female } & Weekends & 54 & 1067216087 & 1430578855 & 0.1272 & 0.1219 \\
& Weekdays & 55 & 734150519 & 978573888 & 0.1154 & 0.1274 \\
\hline \multirow{2}{*}{ male } & Weekends & 95 & 1544937519 & 1986419847 & 0.1461 & 0.1456 \\
& Weekdays & 74 & 694896844 & 1003542127 & 0.1022 & 0.1274 \\
\hline
\end{tabular}

Table 3. Average and standard deviation of area and shape index of user's activity space grouped by days of the week

\begin{tabular}{|c|c|c|}
\hline Gender & std (avg area) & Std (avg SI) \\
\hline Female & 235512921 & 0.0083 \\
Male & 601069525 & 0.0311 \\
\hline
\end{tabular}

Table 4. Effect of weekdays on the activity space of men and women.

\subsection{Correlation between age, days of the week and activity space}

Individuals in all age groups have higher activity space's area and shape index on weekends. It indicates that individuals have more distant and scattered travels and thus active lifestyle on weekends. Moreover, it is observed that the physical shape of activity space also deviates more from a straight line at weekends. The standard deviation of the determinants of the activity space is also higher on weekends, and thus the difference in mobility behavior of individuals is greater (Tables 
5 to 11). As Table 12 shows, the highest value for the standard deviation of the average of activity space determinants belong to those who are 28 to 33 years old and the lowest amount is dedicated to people aged 16 to 21 years and also those over 50 years old. It is well understood from the mentioned results that time has the greatest impact on the movement behavior of people aged 28 to 33 years and have the least effect on people aged 16 to 21 and over 50. Being unemployed and, as a result, having unlimited time for more distant trips among these two groups of people and on the other hand, being employed and therefore having restricted time opportunities for those between 28 and 33 years can be considered as one of the reasons for these results.

\begin{tabular}{|c|c|c|c|c|c|}
\hline Week (age16-21) & No. Users & Avg area & Std area & Avg SI & Std SI \\
\hline Weekends & 13 & 1039875920 & 1110898094 & 0.0993 & 0.092 \\
Weekdays & 13 & 970745566 & 1738028615 & 0.1519 & 0.1127 \\
\hline
\end{tabular}

Table 5. Average and standard deviation of area and shape index of users between 16-21 based on days of the week.

\begin{tabular}{|c|c|c|c|c|c|}
\hline Week (age 22-27) & No. Users & Avg area & Std area & Avg SI & Std SI \\
\hline Weekends & 56 & 1208791969 & 1427396533 & 0.1125 & 0.1079 \\
Weekdays & 56 & 666359556 & 799505045 & 0.0918 & 0.1075 \\
\hline
\end{tabular}

Table 6. Average and standard deviation of area and shape index of users between 22-27 based on days of the week.

\begin{tabular}{|c|c|c|c|c|c|}
\hline Week (age 28-33 ) & No. Users & Avg area & Std area & Avg SI & Std SI \\
\hline Weekends & 46 & 2134567844 & 2656874695 & 0.1439 & 0.1152 \\
Weekdays & 49 & 1005903159 & 1299951503 & 0.1004 & 0.1062 \\
\hline
\end{tabular}

Table 7. Average and standard deviation of area and shape index of users between $28-33$ based on days of the week.

\begin{tabular}{|c|c|c|c|c|c|}
\hline Week (age33-38) & No. Users & Avg area & Std area & Avg SI & Std SI \\
\hline Weekends & 19 & 1182588267 & 1321342023 & 0.1996 & 0.1495 \\
Weekdays & 20 & 663117506 & 726213938 & 0.158 & 0.142 \\
\hline
\end{tabular}

Table 8. Average and standard deviation of area and shape index of users between 33-38 based on days of the week.

\begin{tabular}{|c|c|c|c|c|c|}
\hline Week (age 39-44) & No. Users & Avg area & Std area & Avg SI & Std SI \\
\hline Weekends & 13 & 1154948493 & 1121658229 & 0.1432 & 0.1102 \\
Weekdays & 13 & 608855113 & 572571472 & 0.1355 & 0.1474 \\
\hline
\end{tabular}

Table 9. Average and standard deviation of area and shape index of users between 39-44 based on days of the week.

\begin{tabular}{|c|c|c|c|c|c|}
\hline Week (age 45-50) & No. Users & Avg area & Std area & Avg SI & Std SI \\
\hline Weekends & 2 & 910481240 & 634954914 & 0.0656 & 0.032 \\
Weekdays & 2 & 439812267 & 389629272 & 0.0371 & 0.0234 \\
\hline
\end{tabular}

Table 10. Average and standard deviation of area and shape index of users between 45-50 based on days of the week.

\begin{tabular}{|c|c|c|c|c|c|}
\hline Week (more than 50) & No. Users & Avg area & Std area & Avg SI & Std SI \\
\hline Weekends & 2 & 541437216 & 533857349 & 0.0471 & 0.01 \\
Weekdays & 2 & 296899361 & 299658176 & 0.0854 & 0.0168 \\
\hline
\end{tabular}

Table 11. Average and standard deviation of area and shape index of users more than 50 based on days of the week. 


\begin{tabular}{|c|c|c|}
\hline Age group & Std (avg area) & Std (avg SI) \\
\hline $21-16$ & 48882542 & 0.0372 \\
$27-22$ & 383557638 & 0.0147 \\
$33-28$ & 798086453 & 0.0307 \\
$38-33$ & 367321298 & 0.0294 \\
$44-39$ & 386146332 & 0.0054 \\
$50-45$ & 332813222 & 0.0202 \\
more than 50 & 172914376 & 0.0271 \\
\hline
\end{tabular}

Table 12. Comparison of the effect of weekdays on users' activity space with different age group.

\subsection{Correlation between occupation, days of the week and activity space}

As is shown in tables 13 to 17 , in all working groups the area, shape index and the standard deviation of the activity space determinants were higher on weekends due to the closure of schools, universities, and workplaces which let people have more free time on weekends. Thus, individuals tend to visit more distant places and their destinations are spread out and are not restricted to the pegs of their life. In addition, the result of the comparison of the effect of time on the mobility behavior of individuals with different working status is shown in Table 18. With regard to the value of standard deviation for people in different working groups, it can be concluded that the change in time (days of the week) has the greatest effect on the mobility behavior of employed people and has the least effect on unemployed people. One of the interpretations might be lack of sufficient time opportunity for staff to travel around on weekdays.

\begin{tabular}{|c|c|c|c|c|c|}
\hline Week (working full time) & No Users & Avg area & Std area & Avg SI & Std SI \\
\hline Weekends & 82 & 1661450051 & 1986095364 & 0.1609 & 0.2062 \\
Weekdays & 84 & 841776417 & 934879644 & 0.1347 & 0.2027 \\
\hline
\end{tabular}

Table 13. Average and standard deviation of area and shape index of full-time job users grouped based on weekdays.

\begin{tabular}{|c|c|c|c|c|c|}
\hline Week (working part-time) & No Users & Avg area & Std area & Avg SI & Std SI \\
\hline Weekends & 13 & 2290298195 & 2528344571 & 0.1122 & 0.0759 \\
Weekdays & 14 & 1091894605 & 1719317169 & 0.1273 & 0.1513 \\
\hline
\end{tabular}

Table 14. Average and standard deviation of area and shape index of part-time job users grouped based on weekdays.

\begin{tabular}{|c|c|c|c|c|c|}
\hline Week (not working) & No Users & Avg area & Std area & Avg SI & Std SI \\
\hline Weekends & 7 & 536953830 & 529956734 & 0.1311 & 0.1244 \\
Weekdays & 7 & 383026570 & 358675271 & 0.1015 & 0.0847 \\
\hline
\end{tabular}

Table 15. Average and standard deviation of area and shape index of not working users grouped based on weekdays.

\begin{tabular}{|c|c|c|c|c|c|}
\hline Week (studying full time) & No Users & Avg area & Std area & Avg SI & Std SI \\
\hline Weekends & 41 & 1007158761 & 1097836709 & 0.1083 & 0.1119 \\
Weekdays & 41 & 774265424 & 1103522258 & 0.1094 & 0.1189 \\
\hline
\end{tabular}

Table 16. Average and standard deviation of area and shape index of full time studying users grouped based on weekdays.

\begin{tabular}{|c|c|c|c|c|c|}
\hline Week (housewife) & No Users & Avg area & Std area & Avg SI & Std SI \\
\hline Weekends & 5 & 573722326 & 477361844 & 0.0997 & 0.0087 \\
Weekdays & 5 & 122863208 & 156767262 & 0.098 & 0.1036 \\
\hline
\end{tabular}

Table 17. Average and standard deviation of area and shape index of housewife users grouped based on weekdays. 


\begin{tabular}{|c|c|c|}
\hline Occupation Group & Std (avg area) & Std (avg SI) \\
\hline working full time & 579596785 & 0.0185 \\
working part time & 847399305 & 0.0107 \\
not working & 108843009 & 0.0209 \\
studying full time & 164680458 & 0.0008 \\
housewife & 318805539 & 0.0012 \\
\hline
\end{tabular}

Table 18. Comparison the effect of days of week on users with different occupations.

\section{CONCLUSION}

This paper examines the effect of time (days of the week) on movement characteristics of people and the extent to which it has an impact on different groups of people with a particular same demographic factor (gender, age, and working status). For this, activity space and its determinants (area and shape index) have been deployed to show the extent and the dispersion of movement. The approach was applied on a set the MDC dataset. It was concluded that people have further travels with a more dispersed destination on weekends. Additionally, it has depicted that time has the most effect on the movement behavior of men particularly people aged 28-33 and the least effect on those aged 16-21 and people who are older than 50 years old. We believe that the results of this study have the potential to contribute to the betterment of urban planning, solving social issues and mitigating traffic problems.

\section{References}

Brown, L. A. and E. G. J. G. A. S. B. Moore, Human Geography (1970). "The intra-urban migration process: a perspective." 52(1): 1-13.

Crane, R. J. J. o. t. A. p. a. (2007). "Is there a quiet revolution in women's travel? Revisiting the gender gap in commuting." 73(3): 298-316.

Hagerstrand, T. J. R. S. A. (1970). "What about people in spatial science." 24: 7-21.

Horton, F. E. and D. R. J. E. G. Reynolds (1971). "Effects of urban spatial structure on individual behavior." 47(1): 36-48.

Isaacman, S., R. Becker, R. Cáceres, S. Kobourov, M. Martonosi, J. Rowland and A. Varshavsky (2011). Ranges of human mobility in Los Angeles and New York. 2011 IEEE International Conference on Pervasive Computing and Communications Workshops (PERCOM Workshops), IEEE.

Kiukkonen, N., J. Blom, O. Dousse, D. Gatica-Perez and J. Laurila (2010). "Towards rich mobile phone datasets: Lausanne data collection campaign." Proc. ICPS, Berlin 68.

Laurila, J. K., D. Gatica-Perez, I. Aad, O. Bornet, T.-M.-T. Do, O. Dousse, J. Eberle and M. Miettinen (2012). The mobile data challenge: Big data for mobile computing research.
Lee, N. C., C. Voss, A. D. Frazer, J. A. Hirsch, H. A. McKay and M. Winters (2016). "Does activity space size influence physical activity levels of adolescents?-A GPS study of an urban environment." Preventive medicine reports 3: 75-78.

Luo, F., G. Cao, K. Mulligan and X. J. A. G. Li (2016). "Explore spatiotemporal and demographic characteristics of human mobility via Twitter: A case study of Chicago." 70: 1125 .

Newsome, T. H., W. A. Walcott and P. D. J. T. Smith (1998). "Urban activity spaces: Illustrations and application of a conceptual model for integrating the time and space dimensions." 25(4): 357-377.

Schönfelder, S. and K. W. J. T. p. Axhausen (2003). "Activity spaces: measures of social exclusion?" 10(4): 273-286.

Shi, L., G. Chi, X. Liu and Y. J. A. o. G. Liu (2015). "Human mobility patterns in different communities: a mobile phone data-based social network approach." 21(1): 15-26.

Song, C., Z. Qu, N. Blumm and A.-L. J. S. Barabási (2010). "Limits of predictability in human mobility." 327(5968): 10181021.

Wang, Y., C. Kang, L. M. Bettencourt, Y. Liu and C. Andris (2015). Linked activity spaces: Embedding social networks in urban space. Computational approaches for urban environments, Springer: 313-336.

Yamamoto, T. and R. J. T. Kitamura (1999). "An analysis of time allocation to in-home and out-of-home discretionary activities across working days and non-working days." 26(2): 231-250.

Yuan, Y. (2013). Characterizing human mobility from mobile phone usage, University of California, Santa Barbara.

Yuan, Y., M. Raubal, Y. J. C. Liu, Environment and U. Systems (2012). "Correlating mobile phone usage and travel behavior-A case study of Harbin, China." 36(2): 118-130.

Yuan, Y. and M. J. I. J. o. G. I. S. Raubal (2016). "Analyzing the distribution of human activity space from mobile phone usage: an individual and urban-oriented study." 30(8): 15941621. 